\title{
Use of PARP Inhibitors for Ovarian Cancer
}

\author{
Presented by Deborah K. Armstrong, MD
}

\begin{abstract}
PARP inhibitors have been used to treat numerous diseases, but these agents have been approved the longest for use in ovarian cancer. All trials of PARP inhibitor maintenance in newly diagnosed ovarian cancer are positive for prolonged progression-free survival (PFS), but patients with BRCA mutations consistently derive the most benefit. Testing for homologous recombination deficiency may provide information regarding the degree of PFS benefit. In individuals without a BRCA mutation, PARP inhibition also prolongs PFS after chemotherapy for platinum-sensitive, PARP-naïve disease. As in the up-front setting, patients with BRCA mutations derive the most benefit in these trials. Finally, PARP inhibitors are active as monotherapy in PARP-naïve, BRCA-mutated relapsed disease, with increased activity observed in platinum-sensitive versus platinumresistant disease.

J Natl Compr Canc Netw 2021;19(5.5):636-638 doi: $10.6004 /$ jnccn.2021.5013
\end{abstract}

Although PARP inhibitors have been developed for multiple diseases, they have been approved the longest for treatment of ovarian cancer, and thus offer the most useful data in that indication, according to Deborah K. Armstrong, MD, Director, Breast and Ovarian Surveillance Service, and Professor of Oncology, The Sidney Kimmel Comprehensive Cancer Center at Johns Hopkins. During the NCCN 2021 Virtual Annual Conference, Dr. Armstrong described data from PARP inhibitor trials in patients with newly diagnosed advanced ovarian cancer, reviewed factors that may affect the extent of clinical benefit from PARP inhibitor therapy, and discussed considerations for selecting recurrence therapy in patients whose disease has progressed on or after PARP inhibitor maintenance.

\section{Frontline Maintenance}

The SOLO-1 study enrolled patients with a germline or somatic BRCA mutation and stage III-IV disease who had completed initial therapy. ${ }^{1}$ Patients were also required to be in complete or partial response after platinum-based therapy. Study participants were randomized to receive either the PARP inhibitor olaparib or placebo, and were continued on treatment for 2 years if there was no evidence of disease progression. Results showed a striking difference in median progression-free survival (PFS), which improved from 13.8 months in the placebo group to 56 months in the olaparib group (hazard ratio [HR], 0.33).

"One of the most interesting parts of this study is that after discontinuing olaparib, the survival curves stay separate and maintain benefit out to $>5$ years of followup," said Dr. Armstrong. "Other trials have used longer duration therapy, but our hope is that [olaparib] is nipping disease in the bud early on."

The PAOLA-1 study enrolled patients with newly diagnosed, stage III-IV, high-grade serous/endometrioid ovarian, fallopian tube, or primary peritoneal cancer. $^{2}$ All subjects had to have undergone $\geq 3$ cycles of bevacizumab with their initial chemotherapy. At the end of that initial treatment, patients were randomized to either continue bevacizumab + placebo or bevacizumab + olaparib. Unlike SOLO-1, which selected for patients with a BRCA mutation, PAOLA-1 had broad eligibility. Results demonstrated an improvement in PFS in the intent-to-treat population. Patients treated with bevacizumab alone had a median PFS of 16.6 months, and those treated with bevacizumab + olaparib had a median PFS of 22.1 months (HR, 0.5).

The PRIMA study looked at the PARP inhibitor niraparib in patients with stage III-IV high-grade serous or endometrioid cancer with visible residual disease postsurgery. ${ }^{3}$ All patients had to have experienced a complete or partial response to platinum-based chemotherapy. The primary endpoint showed a median PFS of 8.2 months in the placebo group versus 13.8 months in the niraparib group.

Finally, the VELIA study examined arms combining chemotherapy with a PARP inhibitor in addition to the use of a PARP inhibitor as maintenance therapy. ${ }^{4}$ The study's first arm was placebo + carboplatin/paclitaxel followed by placebo as maintenance. Arm 2 was veliparib + carboplatin/paclitaxel followed by placebo. Arm 3 was veliparib + carboplatin/paclitaxel followed by veliparib maintenance. An improvement in PFS was again observed in patients receiving a PARP inhibitor throughout. Median PFS was 23.5 months for veliparib throughout versus 17.3 months with carboplatin/paclitaxel followed by placebo. 
However, relatively little difference in survival was observed in patients receiving veliparib + chemotherapy.

Unlike prior studies, which had to document a response to initial therapy, patients in the VELIA study were started on the treatment de novo and thus probably included some who would not have responded to therapy, noted Dr. Armstrong. "Because this study may have included a poor prognosis group of patients, the findings may be a little more real-world, and the benefit that is still seen in this group of patients is not just selectively in patients who had responded to initial chemotherapy."

\section{BRCA Mutation Status}

Aside from the SOLO-1 trial, which selected patients based on BRCA mutation status, a similar HR was observed in the other 3 studies, $^{2-4}$ said Dr. Armstrong. Subgroup analysis of BRCA mutation carriers across all 4 studies also showed similarly favorable HRs. "We see the greatest survival benefit in those patients with a $B R C A$ mutation, either somatic or germline," she said.

Although not the same degree of benefit, PARP inhibition also led to improved PFS in patients with BRCA wild-type, according to analysis of the PAOLA-1 and VELIA trials. Furthermore, patients with BRCA wild-type with some measure of homologous recombination deficiency (HRD) had a greater benefit in PFS from PARP inhibitor maintenance than the overall group (18.2 vs 19.6 months in PRIMA $^{3}$; 16.6 vs 28.1 months in PAOLA-1 ${ }^{2}$ ).

Conversely, patients with BRCA wild-type and are homologous recombination proficient are the least likely to benefit from a PARP inhibitor. "There was still some benefit for this population of patients in PRIMA," said Dr. Armstrong, who noted that median PFS went from
5.4 to 8.1 months, ${ }^{3}$ "but the magnitude is different. Also note that patients who are homologous recombination proficient and with BRCA wild-type do worse overall," she added. "Their PFS just with chemotherapy in the placebo arm is worse."

The FDA has approved olaparib for frontline maintenance in patients with germline or somatic BRCA mutations. Niraparib has been approved for use in patients who have experienced a complete or partial response after initial therapy. Olaparib has been approved in combination with bevacizumab for maintenance therapy in those whose cancer is HRD-positive based on the PAOLO-1 study.

\section{Maintenance Post-Recurrence}

For platinum-sensitive, PARP-naïve patients responding to platinum, PARP inhibitor maintenance has also been shown to prolong PFS (Table 1). As in the up-front setting, patients with BRCA mutations also consistently derive the most PFS benefit in these trials.

In the NOVA study, for example, which was enriched for patients with BRCA mutations, median PFS was 5.5 months for placebo versus 21 months for niraparib. ${ }^{5}$ For patients with germline BRCA wild-type, median PFS was 3.9 versus 9.3 months, respectively. Niraparib was the first PARP inhibitor that was FDA approved for the maintenance setting after treatment of recurrent platinum-sensitive disease. "Even without documented BRCA mutation, platinum-sensitive recurrent disease does select for patients who experience benefit from PARP inhibitors," said Dr. Armstrong. "In fact, the benefit in the BRCA wild type is actually a bit more significant than it is in the up-front setting because patients who would develop platinum-resistant recurrence are not included here."

\section{Table 1. Platinum-Sensitive Maintenance}

\begin{tabular}{|c|c|c|c|c|}
\hline & Study $19^{10}$ & $\mathrm{SOLO}^{6}$ & NOVA $^{5}$ & ARIEL $3^{11}$ \\
\hline Patients, N & 265 & 295 & $\begin{array}{l}553 \\
(203 \text { gBRCA, } 350 \text { non-gBRCA) }\end{array}$ & 564 \\
\hline PARP inhibitor & Olaparib & Olaparib & Niraparib & Rucaparib \\
\hline Arms & $\begin{array}{l}\text { 1. Olaparib } \\
\text { 2. Placebo }\end{array}$ & $\begin{array}{l}\text { 1. Olaparib } \\
\text { 2. Placebo }\end{array}$ & $\begin{array}{l}\text { 1. Niraparib } \\
\text { 2. Placebo }\end{array}$ & $\begin{array}{l}\text { 1. Rucaparib } \\
\text { 2. Placebo }\end{array}$ \\
\hline Population & HGS & $\begin{array}{l}\text { HGS/Endometrioid } \\
\text { and BRCAmt }\end{array}$ & HGS & HGS/Endometrioid \\
\hline Primary endpoint & PFS (investigator) & PFS (investigator) & $\begin{array}{l}\text { PFS (BICR) } \\
\text { Independent cohorts: } \\
\text { gBRCAmt } \\
\text { gBRCAwt } \\
\text { Hierarchical HRD } \rightarrow \text { ITT }\end{array}$ & $\begin{array}{l}\text { PFS (investigator) } \\
\text { Hierarchical } \\
\quad \text { BRCAmt } \rightarrow \mathrm{HRD} \rightarrow \mathrm{ITT}\end{array}$ \\
\hline Outcome & $\begin{array}{l}\mathrm{HR}, 0.35 \\
\text { (mPFS, } 4.8 \text { vs } 8.4 \mathrm{mo} \text { ) }\end{array}$ & $\begin{array}{l}\mathrm{HR}, 0.30 \\
\text { (mPFS, } 5.5 \text { vs } 19.1 \mathrm{mo} \text { ) }\end{array}$ & $\begin{array}{l}\text { gBRCAmt: HR, } 0.27 \\
\text { (mPFS, } 5.5 \text { vs } 21.0 \mathrm{mo} \text { ) } \\
\text { gBRCAwt: HR, } 0.45 \\
\text { (mPFS, } 3.9 \text { vs } 9.3 \mathrm{mo} \text { ) }\end{array}$ & $\begin{array}{l}\mathrm{HR}, 0.36 \\
\text { (mPFS, } 5.4 \text { vs } 10.8 \mathrm{mo} \text { ) }\end{array}$ \\
\hline
\end{tabular}

Abbreviations: BICR, blinded, independent, central review; BRCAmt, BRCA mutant; gBRCA, germline BRCA; gBRCAmt, germline BRCA mutation; gBRCAwt, germline BRCA wild-type; HGS, high-grade serous; HR, hazard ratio; HRD, homologous recombination deficiency; ITT, intention to treat; mPFS median progression-free survival; PFS, progression-free survival. 
Similar to the up-front setting, testing for HRD does not identify a patient subpopulation who would experience a PFS benefit from PARP inhibitor maintenance, although it may provide information regarding degree of PFS benefit, Dr. Armstrong added.

SOLO2 was the first trial to show a survival benefit of PARP inhibitor maintenance in BRCA-mutated ovarian cancer. $^{6}$ Median overall survival improved from 38.8 months in the placebo group to 51.7 months in the olaparib group (HR, 0.74). Patients in this trial could not have been previously treated with a PARP inhibitor, so there is no information on whether these benefits would be seen in patients who had previously received PARP inhibitors.

\section{Recurrent Disease}

Active treatment of relapsed disease with PARP inhibitors has also been well tested in BRCA-positive disease, with ARIEL2 and QUADRA studying rucaparib and niraparib, respectively. ${ }^{7,8}$ Although the trials had different criteria regarding prior lines of therapy, objective response rates in this heavily pretreated population were $54 \%$ and $40 \%$, respectively.

In ARIEL2, ${ }^{7}$ which enrolled a patients who were platinum-sensitive, the overall response rate was $80 \%$ for those with $B R C A$ mutations and $29 \%$ for $B R C A$ wild-type with some measure of $\mathrm{HRD}$. The overall response rate was only $10 \%$ in BRCA wild-type with homologous recombination proficiency. In the QUADRA study, ${ }^{8}$ which was platinum agnostic, the overall response rate was $29 \%$ in BRCA-positive disease, $9 \%$ for BRCA wild-type with HRD, and only $3 \%$ for $B R C A$ wild-type.

"Once again, BRCA mutation carriers experience the greatest benefit across the line," said Dr. Armstrong. "The second greatest benefit is seen in patients with $B R C A$ wild-type and some measure of HRD." Furthermore, "these drugs are clearly more active in the platinum-sensitive setting," she added.

\section{Future Directions in PARP Inhibitor Therapy}

Investigators are now currently exploring various combinations of 4 treatment approaches: chemotherapy, bevacizumab, immune checkpoint inhibition, and PARP inhibition.

The triplet combination of olaparib/durvalumab/ bevacizumab, for example, has shown promising efficacy as treatment in the absence of chemotherapy for women with germline $B R C A$ wild-type, platinum-sensitive, relapsed advanced ovarian cancer, with a median PFS of 15 months. ${ }^{9}$ Of note, said Dr. Armstrong, exploratory analysis suggests that the high overall response rate was not driven by differences in genomic instability status. The triple combination is now being tested as part of first-line maintenance treatment in the phase III study.

"The field is getting very complicated," said Dr. Armstrong. "There are a large number of ongoing trials looking at PARP inhibitors with other molecularly targeted agents to overcome resistance to PARP inhibition, either de novo or acquired resistance. It's a very active field of investigation in ovarian cancer."

Disclosures: Dr. Armstrong has disclosed serving as a scientific advisor for AbbVie, Inc.; and receiving grant/research support from AstraZeneca Pharmaceuticals LP, Clovis Oncology, Eisai Inc., Pfizer Inc., and Syndax Pharmaceuticals Inc.

Correspondence: Deborah K. Armstrong, MD, The Sidney Kimmel Comprehensive Cancer Center at Johns Hopkins, 1650 Orleans Street, Room 190, Baltimore, MD 21231. Email: armstde@jhmi.edu

\section{References}

1. Moore K, Colombo N, Scambia G, et al. Maintenance olaparib in patients with newly diagnosed advanced ovarian cancer. N Engl J Med 2018;379:2495-2505.

2. Ray-Coquard I, Pautier P, Pignata $S$, et al. Olaparib plus bevacizumab as first-line maintenance in ovarian cancer. N Engl J Med 2019;381: 2416-2428.

3. González-Martín A, Pothuri B, Vergote I, et al. Niraparib in patients with newly diagnosed advanced ovarian cancer. N Engl J Med 2019;381: 2391-2402.

4. Coleman RL, Fleming GF, Brady MF, et al. Veliparib with first-line chemotherapy and as maintenance therapy in ovarian cancer. N Engl J Med 2019;381:2403-2415.

5. Mirza MR, Monk BJ, Herrstedt J, et al. Niraparib maintenance therapy in platinum-sensitive, recurrent ovarian cancer. N Engl J Med 2016;375:2154-2164.

6. Pujade-Lauraine E, Ledermann JA, Selle F, et al. Olaparib tablets as maintenance therapy in patients with platinum-sensitive, relapsed ovarian cancer and a BRCA1/2 mutation (SOLO2/ENGOT-Ov21): a double-blind, randomised, placebo-controlled, phase 3 trial. Lancet Oncol 2017;18:1274-1284.

7. Swisher EM, Lin KK, Oza AM, et al. Rucaparib in relapsed, platinum-sensitive high-grade ovarian carcinoma (ARIEL2 part 1): an international, multicentre, open-label, phase 2 trial. Lancet Oncol 2017;18:75-87.

8. Moore KN, Secord AA, Geller MA, et al. Niraparib monotherapy for lateline treatment of ovarian cancer (QUADRA): a multicentre, open-label, single-arm, phase 2 trial. Lancet Oncol 2019;20:636-648.

9. Drew Y, Penson RT, O'Malley DM, et al. Phase II study of olaparib plus durvalumab and bevacizumab (MEDIOLA): initial results in patients with non-germline BRCA-mutated platinum sensitive relapsed ovarian cancer [abstract]. Ann Oncol 2020;31(Suppl 4):S615-616. Abstract $814 \mathrm{MO}$

10. Ledermann J, Harter P, Gourley C, et al. Olaparib maintenance therapy in platinum-sensitive relapsed ovarian cancer. N Engl J Med 2012;366: 1382-1392.

11. Coleman RL, Oza AM, Lorusso D, et al. Rucaparib maintenance treatment for recurrent ovarian carcinoma after response to platinum therapy (ARIEL3): a randomised, double-blind, placebo-controlled, phase 3 trial. Lancet 2017:390:1949-1961. 